Copyright (C) 2014 by Academic Publishing House Researcher

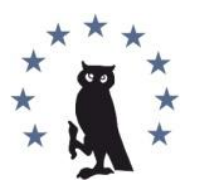

Published in the Russian Federation

European Researcher

Has been issued since 2010.

ISSN 2219-8229

E-ISSN 2224-0136

Vol. 86, No. 11-1, pp. 1901-1909, 2014

DOI: 10.13187/er.2014.86.1901

www.erjournal.ru

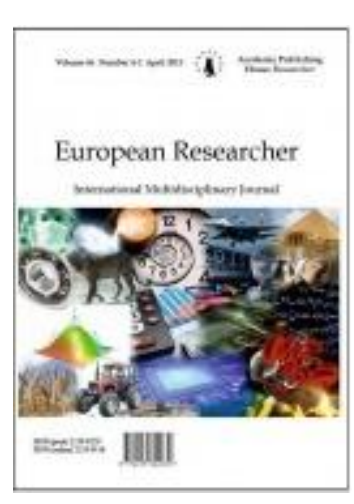

UDC 004.041

\title{
Dichotomic Assessment of Information Situations and Information Superiority
}

\author{
Victor Ya. Tsvetkov \\ Moscow State Technical University of Radio Engineering, Electronics and Automation MSTU MIREA, \\ Russian Federation \\ Adviser \\ E-mail: cvj2@mail.ru
}

\section{Abstract}

The article describes a methodology for assessing information situations and determining information characteristics between objects - information superiority. The author brings to light the concept of information superiority for static and dynamic information situations; applies dichotomic analysis for exploring information situations and information superiority; describes static and dynamic information situations; brings to light the concept of informing and information states; illustrates three types of assessing information states; describes various information situations: "by the degree of being informed", "by goal achievement", "by flows", and "by interaction"; provides formulas for assessing states and coefficients for assessing these states; illustrates that the proposed methodology helps assess the competitiveness of objects and resource support for them.

Keywords: information; information situation; information flows; informing; information interaction; information state; information superiority; resource support; information relations.

\section{Введение}

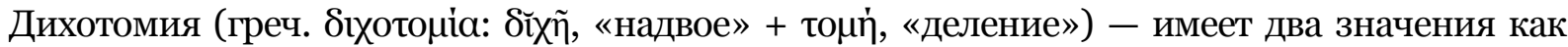
свойство и как процесс или метод. Как свойство дихотомия означает раздвоенность. Как метод дихотомия обозначает процесс последовательного деления объекта исследования на две части, не связанные между собой [1]. Дихотомия обозначает также процесс логического деления класса на подклассы, который состоит в том, что делимое понятие полностью делится на два взаимоисключающих понятия. Можно говорить о полной дихотомии и частичной дихотомии. Полная дихотомия с лингвистических позиций сохраняет объем понятия, а с позиций семантики сохраняет все существенные признаки при делении. Такой подход позволяет формировать структурную модель [2], оценивать сложность [3] или структурировать информационные ресурсы [4].

Частичная дихотомия может не сохранять объем понятия или исключать некоторые признаки. Главной ее целью является исследование двух важных факторов, безотносительно к другим. Она используется при исследовании категориальных пар [5], таких как: «необходимое свободное», «внутреннее - внешнее», «информационные потребности - материальноэнергетические потребности» и т.п.

Преимуществом дихотомии является простота. При полной дихотомии человек имеет дело лишь двумя классами, которые исчерпывают объём делимого понятия. Если объект деления $O$ 
полностью делится на два класса $a$ и $b$, то это очень удобно. При этом одним из видовых отличий служит само основание деления $a$, а другим - его логическое дополнение $b$.

Пример: «человек» $\rightarrow$ «мужчины»; «женщины». $O$ - человек, $a-$ мужчины, $b-$ женщины. Такое деление происходит в рамках одной категории. Здесь имеет место «целое» и результаты его деления: основание и логическое дополнение.

Однако при делении объёма понятия с использование отрицания «не» на два противоречащих понятия может быть неопределённой та его часть (логическое дополнение), к которой относится частица «не». Пример: «мебель» $\rightarrow$ «стол» и «не стол». $O$ - мебель, $a-c m o л$. В часть не $a$ - попадают объекты других категорий, например: животное, человек, автомобиль, самолет, планета Земля. Следовательно, при делении с использованием отрицания необходимо вводить дополнительное условие, ограничивающее область отрицания.

Особое место при дихотомическом делении занимают оппозиционные переменные, которые противоречат друг другу [6, 7]. Например, «достоинства - недостатки», «прибыль убытки», «ускорение - торможение» и т.д. Часто оппозиционные переменные показывают крайние стороны и исключают промежуточные значения. В этом случае идет речь о частичном дихотомическом делении, поскольку одна оппозиционная переменная не является полным дополнением другой до целого и объем понятия уменьшается.

\section{Методика}

Методика оценки основана на введении базовых понятий, и проведении качественных и количественных оценок, связанных с дихотомическим делением. То есть по существу методика опирается на синтез качественного и количественного анализа. Применяем следующие базовые понятия: «информационная ситуация» [8], «информационное состояние» [9], «общее состояние», «информированность». Данная оценка применима в равной степени для объекта или субъекта [10], но в дальнейшем будем применять термин объект, имея в виду возможность его использования для обозначения «субъекта». Информационная ситуация есть информационная модель, которая характеризует информационное окружение [11] объекта анализа или его локальную информационную среду [12]. Это некая пассивная информационная модель, в которой находится анализируемый объект или субъект.

Информационная ситуация может содержать в себе множество объектов, являясь по отношению к ним информационной средой [12]. Это дает основание проводить сравнение объектов и их состояний в данной информационной ситуации. Информационная ситуация может содержать в себе множество состояний для разных объектов. Это дает основание проводить сравнение объектов по состояниям, в которых они находятся.

Необходимо различать понятия “информационное состояние” объекта и “общее состояние” объекта. Информационное состояние объекта или субъекта определяется как набор параметров для определенной информационной ситуации. Информационное состояние объекта $A$ в момент времени $t_{i}$ можно рассматривать как информационную модель, оцениваемую относительно:

- состояния объекта $A$ в предыдущий момент времени $t_{i-1}$;

- другой информационной модели, которой является другой объект $B$;

- цели $T$, которую стремиться достичь объект $A$.

Информационное состояние является сравнительной характеристикой. В реальности оно оценивается только по информационными характеристиками, в частности, по количеству и качеству информационных ресурсов. Информационное состояние оценивается частью набора параметров, которые входят в описание информационной ситуации. Для одной информационной ситуации может быть много информационных состояний объекта $A$. Информационное состояние может оцениваться относительно любого другого объекта в заданной информационной ситуации или относительно заданной цели объекта.

Модель общего состояния рассматривается тогда, когда объект начинает реализовывать своё предназначение и удовлетворять материальные, энергетические и информационные потребности. Общее состояние объекта определяется полным набором параметров, включая те которые не входят в описание информационной ситуации. Общее состояние объекта или субъекта описывается большим числом параметров, по сравнению с числом параметров, описывающих информационное состояние. К числу параметров, описывающих общее состояние, относятся характеристики: информационных, финансовых, энергетических и прочих 
- ресурсов. В дальнейшем будем употреблять для сокращения термин «информация», который будет обозначать информационные ресурсы.

В аспекте цели для объекта $A$ необходимо различать понятия: “фактическое информационное состояние" и “необходимее информационное состояние”, которое должно быть достигнуто для достижения цели или решения задачи. Неравенство “фактического” и “необходимого" информационных состояний и означает наличие дихотомии.

Информированность в абсолютной оценке обозначает реальное количество и качество информационных ресурсов, которыми обладает объект $A$. Такая информированность объекта $A$ характеризует его фактическое информационное состояние. Она называется «ресурсная информированность» поскольку характеризует ресурсы объекта.

Информированность в относительной оценке означает сравнение информирования с тем уровнем или состоянием, которое необходимо для достижения поставленных целей. Такая информированность характеризует необходимое информационное состояние. Она называется информированность «по цели», поскольку характеризует возможность достижения цели.

Информированность в относительной оценке можно оценить также при сравнении информационных ресурсов разных объектов. Она называется информированность «по объекту сравнения».

Информационные ситуации $I S\left(P_{1}, P_{2}(t)\right)$ разделяют на статические и динамические [13]. Статическая информационная ситуация описывается параметрами, которые не изменяются с течением времени $I S\left(P_{1}\right)$. Динамическая информационная ситуация описывается параметрами, которые изменяются с течением времени и для которых известна зависимость изменения от времени $I S\left(P_{1}, P_{2}(t)\right)$. Для динамической информационной ситуации состояние объекта $A$ в предыдущий момент времени $t_{i-1}$; отличается от состояния в текущий момент времени и в будущий момент времени. Дихотомия состояний или ситуаций будет служить характеристикой динамики состояния или ситуации. Дихотомией в данном случае будем называть различие в состояниях для одного объекта в разные моменты времени.

\section{Результаты}

Рассмотрим информационную ситуацию, в которой может проводиться сравнение двух объектов. Для обозначения неравенства состояний объектов можно использовать математический знак «неравенство». Это позволяет создать простое описание отношения объектов по информированности.

$$
\begin{aligned}
& A(I)>B(I)(1) \\
& A(I)<B(I)(2)
\end{aligned}
$$

Выражения (1) и (2) определяют неравенство между обозначенными $A, B$ - объектами по информированности (I). Данная информационная ситуация называется ситуацией «ситуация отношений объектов по информированности».

Выражение (1) интерпретируется следующим образом. Фактическая информированность объекта $A$ больше, чем информированность объекта $B$. Выражение (2) интерпретируется с противоположным смыслом. Информированность объекта $B$ больше, чем информированность объекта $A$. Выражение (1) говорит об информационном превосходстве объекта $A$ над $B$. Выражение (2) говорит об информационном превосходстве объекта $B$ над $A$.

Определение, информационным превосходством по состоянию называется информационная ситуация отражающая большую информированность одного объекта в сравнении с другим. Если знак неравенства в (1), (2) заменить на знак равенства, то будет иметь место «информационное соответствие» [14] по информированности.

В интерпретации выражений (1) и (2) проводилось сравнение между объектами $A$ и $B$. Такое сравнение можно определить как сравнение по объектам. Если объектов не два, а N, то можно провести $\mathrm{N}$ парных сравнений и используя методы любой теории сравнения, например теории предпочтений [15], построить систему предпочтительности для $\mathrm{N}$ объектов и ранжировать их по выбранному критерию. Это определяет возможность сравнения по информированности для любого числа объектов.

Если ввести понятие целевой информации $I_{T}$, которое обозначает информацию, необходимую для достижения цели $T$, то можно ввести характеристику еще одной 
информационной ситуации

$$
\begin{gathered}
A(I)>T(I)(3) \\
A(I)<T(I)(4)
\end{gathered}
$$

Выражение (3) интерпретируется следующим образом. Фактическая информированность объекта $A$ достаточная для достижения цели. Выражение (4) интерпретируется с противоположным смыслом. Фактическая информированность объекта $A$ не достаточна для достижения цели. Данная информационная ситуация называется «ситуация по достижению цели». При этом количество объектов может быть любым. Если объектов N, то можно провести $\mathrm{N}$ парных сравнений и построить таблицу объектов, способных достичь цель, и таблицу объектов, неспособных достичь цель.

Первый вариант сравнения “по объектам" (1)-(2), позволяет оценить конкурентоспособность объектов данной совокупности между собой на рынке или в иной ситуации взаимодействия. Второй вариант сравнения (3)-(4) дает основание принятия решений по изменению ситуации с обеспечением ресурсами определенных объектов.

Выражения (1), (2), (3), (4) характеризуют статические информационные ситуации. Определим коэффициент целевой информированности $K_{I}$ как

$$
K_{I}=I_{F} / I_{T}(5)
$$

Где $I_{T}$ - количество информации, необходимое для достижения цели, $I_{F}-$ количество информации которым фактически обладает объект или субъект. Количеством информации обозначаем содержательную составляющую, а не информационный объем. Этот коэффициент имеет значение от о до 1.

Возможна информационная ситуация, при которой к объекту или субъекту поступают информационные потоки из разных внешних источников. Эта ситуация возможна при наличии разных источников информации и потоков разной интенсивности. Такую ситуацию называют потоковой информационной ситуацией. Информационные потоки разной интенсивности и разного качества изменяют информационные состояния объектов.

$$
F_{S 1} \rightarrow I_{B}>F_{S 2} \rightarrow I_{A}(6)
$$

Выражение (6) интерпретируется следующим образом. Информационный поток $F_{S 1}$, направленный к объекту $B$, превосходит информационный поток $F_{S 2}$, направленный к объекту $A$, что создает информационное превосходство по информационным потокам.

Определение, информационным превосходством по информационным потокам называется информационная ситуация отражающая большую интенсивность информационного потока для одного объекта в сравнении с другим.

Такая информационная ситуация называется потоковой информационной ситуацией. Данная информационная ситуация является процессуальной, поскольку характеризует процесс. Определим относительный коэффициент информационного потока $K_{F B}$ к объекту $B$ как

$$
K_{F B}=F_{S 1} /\left(F_{S 1}+F_{S 2}\right)(7)
$$

Определим относительный коэффициент информационного потока $K_{F A}$ к объекту $A$ как

$$
K_{F A}=F_{S 2} /\left(F_{S 1}+F_{S 2}\right)(8)
$$

Относительные коэффициенты информационного потока $K_{F A}$ и $K_{F B}$ имеют значения от о до 1. Особенностью потоковой информационной ситуации, приведенной в выражении (6), является то, что знак неравенства может быть заменен знаком равенства. В этом случае будет информационное соответствие [14] по потокам.

Частным случаем информационного превосходства по потокам является ситуация, связанная с взаимным информационным обменом между двумя объектами. Она возникает при наличии взаимных разнонаправленных информационных потоков. Потоки выполняют пассивную роль информирования объектов. Такая информационная ситуация является процессуальной, поскольку обусловлена процессом. Информационный обмен изменяет 
количество информации объектов и также может создавать информационное превосходство по обмену информацией.

$$
F_{A}: I_{A} \rightarrow I_{B}>F_{B}: I_{B} \rightarrow I_{A}(9)
$$

Выражение (9) интерпретируется следующим образом. Информационный поток $F_{A}$, по направлению от $A$ к $B$, превосходит информационный поток $F_{B}$ по направлению от $B$ к $A$, что приводит к информационному превосходству по обмену информацией. Определим коэффициент информационного обмена $K A_{E X C}$ от источника $A$ к источнику $B$ как

$$
K A_{E X C}=F_{A} /\left(F_{B}+F_{A}\right)(10)
$$

В противоположную сторону

$$
K B_{E X C}=F_{B} /\left(F_{B}+F_{A}\right)(11)
$$

Здесь $F_{A}$ - интенсивность потока от $A$ к $B, F_{B}$ - интенсивность потока от $B$ к $A$. Коэффициенты $K A_{E X C}$ и $K B_{E X C}$ Можно сравнивать между собой. Они нормированы от о до 1.

Потоковая информационная ситуация меняет информированность объекта и может привести к информационной асимметрии [16]. В то же время, не всякая информационная потоковая ситуация приводит к информационной асимметрии. Это зависит от фактического уровня информированности, определяемого выражениями (1) и (2). Например, при наличии семантического разрыва [17], направление потока может либо уменьшать, либо увеличивать семантический разрыв. Следовательно, информационная потоковая ситуация может служить средством уменышения информационного превосходства по состоянию.

Возможно информационное превосходство, которое возникает в ходе информационного взаимодействия [18]. Взаимодействие в отличие от обмена информацией является активным процессом [19]. Обмен информацией меняет информированность объектов и воздействует только на информационное состояние. Взаимодействие воздействует на общее состояние объекта и может его менять. Ситуация информационного взаимодействия характеризуется потоками, которые направлены на изменение общего состояния объекта.

$$
\operatorname{Int}_{A}: I_{A} \rightarrow I_{B}>\operatorname{Int}_{B}: I_{B} \rightarrow I_{A}(12)
$$

Выражение (12) интерпретируется следующим образом. Информационное воздействие $\operatorname{Int}_{A}$ объекта $A$, превосходит информационное воздействие $\operatorname{Int}_{B}$ объекта $B$, что создает информационное превосходство. Эта информационная ситуация является процессуальной, поскольку характеризует процесс. Однако данная информационная ситуация может быть сбалансированной. В этом случае говорят о информационной согласованности или информационном соответствии объектов $A$ и $B$ [14]. Определим коэффициент информационного взаимодействия $K_{I N T}$ от источника $A$ к источнику $B$ как

$$
K A_{I N T}=\operatorname{Int}_{A} /\left(\operatorname{Int}_{A}+\operatorname{Int}_{B}\right)(13)
$$

Частным случаем выражения (12) является ситуация, когда $B$ обозначает объект управления, а Int ${ }_{B}$ интенсивность воздействия, необходимую для достижения цели объектов $B$. В этом случае будем иметь две возможных ситуации

$$
\begin{aligned}
& \operatorname{Int}_{A}: I_{A} \rightarrow I_{B} \geq \text { Int }_{B}: \text { (14) } \\
& \operatorname{Int}_{A}: I_{A} \rightarrow I_{B}<\text { Int }_{B}:(15)
\end{aligned}
$$

Выражение (14) интерпретируется следующим образом. Информационное воздействие (управляющее воздействие) $\operatorname{Int}_{A}$ объекта $A$, достаточно для достижения цели объектом или для изменения его состояния $B$. 
Выражение (15) интерпретируется следующим образом. Информационное воздействие (управляющее воздействие) $\operatorname{Int}_{A}$ объекта $A$, недостаточно для достижения цели объектом $B$ или для изменения его состояния.

Возможна ситуация информационного воздействия, при которой разные объекты независимо воздействуют на $A$ и $B$. Такая ситуация называется “информационная ситуация независимых воздействий”. Аналитическая модель информационной ситуации при внешних воздействиях $(E x A)$ на объекты с целью изменения их состояний, имеет следующий вид.

$$
E x A_{1} \rightarrow I_{B}>E x A_{2} \rightarrow I_{A}(16)
$$

Выражение (16) интерпретируется так: внешнее информационное воздействие $E x A_{1}$ на объект $B$ создает его информированность $I_{B}$ и превосходит внешнее информационное воздействие $E x A_{2}$ на объект $A$, которое создает информированность $I_{A}$. Это создает информационное превосходство объекта $B$ над объектом $A$. Такая информационная ситуация является процессуальной, поскольку обусловлена процессом. Выражение (16) может сигнализировать о том, что ресурсное обеспечение объекта $B$ превосходит ресурсное обеспечение объекта $A$.

Определим коэффициент относительного информационного воздействия $K A_{A}$ на объект $A$ как

$$
K A_{A}=E x A_{2} /\left(E x A_{2}+E x A_{1}\right)(17)
$$

Определим коэффициент относительного информационного воздействия $K B_{A}$ на объект $B$ как

$$
K B_{A}=E x A_{1} /\left(E x A_{2}+E x A_{1}\right)(18)
$$

Возможна информационная ситуация, которая возникает в ходе ситуационного анализа [20] Пусть объект $A$ находится в ситуации $S_{1}$, которая характеризуется набором параметров $P_{1}$. Объект В находится в ситуации $S_{2}$, которая характеризуется набором параметров $P_{2}$. Используя теорию предпочтений [15] или другой критерий [21] можно (условно) придти к выводу, что $P_{1}$ предпочтительнее $P_{2}$,

$$
P_{1} \Rightarrow P_{2}(19)
$$

Выражение (19) говорить о том, что $S_{1}$ предпочтительнее $S_{2}$, что влечет наличие информационного превосходства в ситуациях между $A$ и $B$. Такая информационная ситуация предпочтительности сигнализирует о том, что объект $A$ является более конкурентоспособным в сравнении с объектом $B$.. Если считать $B$ целью, которую должен достичь объект $A$, то выражение (19) говорит о том, что объект $A$ находится в ситуации, позволяющей достичь цель $B$. При замене направления предпочтительности в выражении (19) на противоположное, возможна иная интерпретация выражения (19). Объект $A$ находится в ситуации, которая не позволяет достичь цели $B$.

\section{Обсуждение}

Информационное превосходство является сравнительной характеристикой. При этом возможна двойственность сравнения между подобными объектами и между объектом и достигаемой им цели. Рассмотренные методы оценки включают качественный и количественный анализ.

Выражения (1), (2), (3), (4), (6), (9), (12), (14), (15), (16), (19) основаны на качественноколичественных оценках. Они требуют экспертных оценок. При этом оценки имеют большей частью сравнительный характер. Это означает, что при изменении числа параметров, применяемых для оценивания, результат может меняться. Оценки такого рода имеет область истинности только на группе сравниваемых параметров.

Выражения (5), (7), (8), (10), (11), (13), (17), (18) используют количественные меры, что дает 
возможность получения объективной количественной оценки. Особенностью этих выражений в том, что все количественные оценки в них нормированы между о и 1. Это создает условия не только для получения оценок, но применения методов статистического анализа и методов теории вероятности для оценивания гипотез первого и второго рода. Однако вес или значимость количественных оценок (5), (7), (8), (10), (11), (13), (17), (18) зависят от качественных оценок, получаемых в выражениях (1), (2), (3), (4), (6), (9), (12), (14), (15), (16), (19). Основная проблема оценки информационных ресурсов при их большом количестве это сложность [3]. Сложность оценки информационных ресурсов или сложность информационной ситуации [8] приводит к необходимости упрощения, которое каждый эксперт делает с произволом. Это создает условия для искажения ситуации и получения правдоподобной, но не истинной оценки. Такое качественное оценивание может сыграть негативную роль при недобросовестном экспертном оценивании или тенденциозном экспертном оценивании.

Информационное превосходство может быть естественным и искусственным. Например, при обучении, естественное информационное превосходство характерно для всех преподавателей. Потоковое действие и информационное взаимодействие между преподавателем и учеником устраняет это превосходство. В этом случае осознание превосходства мотивирует субъект к его устранению.

\section{Заключение}

В данной работе нет разграничения между объектом и субъектом. Это означает, что результаты применимы для оценки информационного превосходства как между информационными системами, так и субъектами. Для оценки информационного превосходства и его устранения применимы аналитические выражения, приведенные в данной работе. На основе приведенных выражений и методов возможно оценивание конкурентоспособности группы объектов между собой. Результаты работы позволяют получать характеристику "информационное превосходство" объекта перед другим объектом. Результаты работы позволяют оценить возможность достижения цели объектом "по ситуации" или “по информированности”. Результаты работы дают возможность оценивать такой показатель как “ ресурсное обеспечение объекта”.

\section{Примечания:}

1. Tsvetkov V.Ya. Dichotomous Systemic Analysis. Life Science Journal 2014. 11(6). pp. 586-590.

2. Елсуков П.Ю. Формирование структурной модели при управлении энергосбережением // Вестник МГТУ МИРЭА. 2014. № 3 (4). с. 135-145.

3. Ожерельева Т.А. Сложность информационных ресурсов. // Современные наукоемкие технологии. 2014. №4. с. 80-85.

4. Тымченко Е.В. Структуризация информационных образовательных ресурсов // Управление образованием: теория и практика. 2014. № 3.(15). с. 181-188.

5. Соловьёв И.В. Сложная организационно-техническая система как инструмент исследования искусственных антропогенных систем // Дистанционное и виртуальное обучение. №1. 2014. с. 5-23.

6. Tsvetkov V.Ya. Opposition Variables as a Tool of Qualitative Analysis // World Applied Sciences Journal. 2014. 30 (11). p. 1703-1706.

7. Цветков В.Я. Использование оппозиционных переменных для анализа качества образовательных услуг // Современные наукоёмкие технологии. 2008. № 1. с. 62-64.

8. Tsvetkov V.Ya. Information Situation and Information Position as a Management Tool // European Researcher, 2012, Vol.(36), № 12-1, p. 2166-2170.

9. Traum D.R., Larsson S. The information state approach to dialogue management // Current and new directions in discourse and dialogue. Springer Netherlands, 2003. C. 325-353.

10. Соловьёв И.В. О субъекте и объекте инфосферы // Перспективы науки и образования, 2013. №5. с. 14-18.

11. Цветков В.Я., Чехарин Е.Е. Окружение информационных единиц // Вестник МГТУ МИРЭА. 2014. № 2 (3). с. 36-42.

12. Ожерельева Т.А. Об отношении понятий информационное пространство, информационное поле, информационная среда и семантическое окружение // Международный журнал прикладных и фундаментальных исследований. 2014. №10. с. 21-24. 
13. Цветков В.Я., Шорыгин С.М. Динамическая информационная ситуация преодоления противоракетной обороны // Вестник МГТУ МИРЭА. 2014. № 3 (4). с. 85-100.

14. Mikkelsen G., Aasly J. Concordance of information in parallel electronic and paper based patient records // International journal of medical informatics. 2001. №63 (3). p. 123-131.

15. Цветков В.Я. Основы теории предпочтений. М.: МаксПресс, 2004. 48 с.

16. Aboody D., Lev B. Information asymmetry, R\&D, and insider gains //The journal of Finance. 2000. T. 55. №. 6. C. 2747-2766.

17. Tsvetkov V.Ya. Information Interaction as a Mechanism of Semantic Gap Elimination. // European Researcher. 2014. Vol.(45), №4-1, pp. 782-786.

18. Shedroff N. Information interaction design: A unified field theory of design. // Information design, 1999. p. 267-292.

19. Tsvetkov V.Ya. Information interaction // European Researcher, 2013, Vol.(62), № 11-1, p. 2573-2577.

20. Цветков В.Я. Ситуационное моделирование в геоинформатике // Информационные технологии. 2014. №6. с. 64-69.

21. Saaty T.L. Decision making with the analytic hierarchy process International journal of services sciences. 2008. №1 (1). P. 83-98.

\section{Referenses:}

1. Tsvetkov V.Ya. Dichotomous Systemic Analysis. Life Science Journal 2014. 11 (6). pp. 586-590.

2. Elsukov P.Yu. Formation of the structural model in the management of energy saving //

MSTU MIREA HERALD 2014. № 3 (4). pp. 135-145.

3. Ozhereleva T.A. The complexity of information resources. // Modern high technologies. 2014. №4. p. 80-85.

4. Tymchenko E.V. Structuring information educational resources // Education Management: Theory and Practice. 2014. № 3 (15). p. 181-188.

5. Soloviev I.V. Complex organizational and technical system as a research tool of artificial human systems // distance and virtual learning. №1. 2014. p. 5-23.

6. Tsvetkov V.Ya. Opposition Variables as a Tool of Qualitative Analysis // World Applied Sciences Journal. 2014. 30 (11). pp. 1703-1706.

7. Tsvetkov V.Ya. Using opposition variables to analyze the quality of educational services // Modern high technologies. 2008. №1. p. 62-64.

8. Tsvetkov V.Ya. Information Situation and Information Position as a Management Tool // European Researcher, 2012, Vol.(36), № 12-1, pp. 2166-2170.

9. Traum D.R., Larsson S. The information state approach to dialogue management // Current and new directions in discourse and dialogue. Springer Netherlands. 2003. C. 325-353.

10. Soloviev I.V. On the subject and object of the infosphere // Perspectives of Science and Education, 2013. №5. pp. 14-18.

11. Tsvetkov V.Ya., Cheharin E.E. Setting information units // MSTU MIREA HERALD. 2014. № 2 (3). pp. 36-42.

12. Ozhereleva T.A. On the relation of the notion of information space, information field, information environment and semantic environment // International Journal of applied and fundamental research. 2014. №10. p. 21-24.

13. Tsvetkov V.Ya, Shorygin S.M. Dynamic information situation overcome missile defense // MSTU MIREA HERALD. 2014. № 3 (4). c. 85-100.

14. Mikkelsen G., Aasly J. Concordance of information in parallel electronic and paper based patient records // International journal of medical informatics. 2001. №63 (3). p. 123-131.

15 Tsvetkov V.Ya. Fundamentals of the theory of preference. Moscow, MaksPress, 2004. 48 p.

16. Aboody D., Lev B. Information asymmetry, R\&D, and insider gains // The journal of Finance. 2000. T. 55. №. 6. C. 2747-2766.

17. Tsvetkov V.Ya. Information Interaction as a Mechanism of Semantic Gap Elimination. // European Researcher. 2014. Vol.(45), №4-1, pp. 782-786.

18. Shedroff N. Information interaction design: A unified field theory of design. // Information design, 1999. p. 267-292.

19. Tsvetkov V.Ya. Information interaction // European Researcher, 2013, Vol.(62), № 11-1, p. 2573-2577. 
20. Tsvetkov V.Ya. Situational modeling in Geoinformatics // Information Technology. 2014. №6. pp. 64-69.

21. Saaty T.L. Decision making with the analytic hierarchy process International journal of services sciences. 2008. №1 (1). P. 83-98.

\section{УДК 004.041}

\section{Дихотомическая оценка информационной ситуации и информационного превосходства}

\section{Виктор Яковлевич Цветков}

Московский государственный технический университет радиотехники, электроники и автоматики МГТУ МИРЭА, Российская Федерация

Доктор технических наук профессор.

Советник

E-mail: cvj2@mail.ru

Аннотация. Статья описывает методику оценки информационных ситуаций и определения информационной характеристики между объектами - информационное превосходство. Статья раскрывает понятие информационного превосходства для статической и динамической информационной ситуации. Применяется дихотомический анализ для исследования информационной ситуации и информационного превосходства. Описаны статические и динамические информационные ситуации. Раскрывается понятие информирования и информационного состояния. Показаны три вида оценки информационного состояния. Описаны разные информационные ситуации: «по информированности», «по достижению цели», «по потокам», «по взаимодействию». Даны формулы оценки состояний и коэффициентов оценки этих состояний. Показано, что предложенная методика позволяет оценивать конкурентоспособность объектов и их ресурсное обеспечение.

Ключевые слова: информация; информационная ситуация; информационные потоки; информирование; информационное взаимодействие; информационное состояние; информационное превосходство; ресурсное обеспечение; информационные отношения. 